1 International Journal of Image and Graphics Vol. 5, No. 1 (2005) 1-29

3 (c) World Scientific Publishing Company

\title{
IMAGE WATERMARKING RESISTANT TO COMBINED GEOMETRIC AND REMOVAL ATTACKS
}

\author{
CHOONG-HOON LEE* ${ }^{*}$ and HEUNG-KYU LEE ${ }^{\dagger}$ \\ Department of Computer Science and \\ Advanced Information Technology Research Center (AITrc), \\ Korea Advanced Institute of Science and Technology (KAIST), \\ Guseong-Dong, Yuseong-Gu, Daejon, 305-701, Korea

In this paper, we propose an improved autocorrelation function (ACF)-based watermarking that is robust against combined geometric and removal attacks. ACF-based watermarking is known to be effective against geometric attacks. In this watermarking scheme, both the embedded watermark and autocorrelation peaks of the watermark should survive a variety of attacks. However, the autocorrelation peaks are not strong enough. The proposed method improves the robustness of ACF-based watermarking by enhancing the strength of the autocorrelation peaks. Instead of the simple addition of a watermark to an image, the proposed watermark embedder uses the information of an original image more actively during the embedding process, so that the marked image has strong autocorrelation peaks. Experimental results show that the proposed scheme provides better robustness than conventional ACF-based watermarking against a variety of combined geometric-removal attacks.

Keywords: Copyright protection; image watermarking; geometric attack; ACF-based watermarking; watermarking with side information.

\section{Introduction}

Digital multimedia markets have grown rapidly over the last decade. Movies, music and other multimedia contents are digitized and distributed via networks. Digital media have many advantages over analog media. They can be copied without loss of fidelity and edited easily. However, these advantages render it easier to make illegal copies or forgeries of copyrighted media. 
In the past few years, digital watermarking has been considered as a possible solution to the problem of protecting copyrighted digital media. ${ }^{1,2}$ Digital watermarking is a process of hiding copyright information in the multimedia data to be protected. If it is to provide efficient copyright protection, digital watermarking must satisfy two requirements: unobtrusiveness and robustness. Unobtrusiveness means that watermark embedding should not affect the quality of the data. Robustness means that embedded watermarks should be able to be detected reliably, even after watermarking attacks.

Geometric attacks are considered to be very strong in the watermarking world. They effectively disable the watermarking system with little degradation of image quality. Geometric attacks do not remove or attenuate an embedded watermark, but prevent its detection by changing its position, angle and scale. In spread-spectrum watermarking, ${ }^{3,15}$ which is the most prevalent watermarking technique, synchronization between an embedded watermark and a reference watermark is very important for watermark detection. If an embedded mark is distorted geometrically, a watermark detector considers it to be a totally different signal from the reference mark, even if it is a correct watermark. In this case, detection of the watermark is impossible without first reversing the applied geometric distortion.

One approach to combat geometric attacks is to embed a watermark into a geometric attack invariant domain. The Fourier-Mellin transform based method ${ }^{5,10}$ adopts this approach. In this method, an image is transformed into a geometric attack invariant domain by the Fourier-Mellin transform and a watermark is embedded. The problem with this method is that perfect inversion of the Fourier-Mellin transform is impossible. Thus, interpolation in the Fourier domain is necessary during the inversion, and the resulting image quality is very poor.

Feature-based watermarking is another watermarking scheme that resists geometric attacks. ${ }^{13,14}$ This method uses geometric distortion-invariant features such as edges or corners, for watermark embedding. A target image is segmented using the distortion-invariant features, then each segment is geometrically normalized and a watermark is embedded into the normalized segments. This method requires a stable feature extractor that always finds the same features, even when the image has been processed, for example by an image filtering, subsequent to the embedding of the watermark.

Template-based watermarking also addresses geometric attacks. ${ }^{4,6}$ In this scheme, a template is embedded into a host image in addition to the embedding of the watermark. The template does not contain copyright information in itself. It is just a special pattern of peaks that are used for the estimation of the geometric attacks. It is embedded into the Fourier transform domain of a host image. During watermark detection, the applied geometric attacks are estimated and reversed by inspecting the template pattern. With this method, watermark detection will fail if there is any failure in either of the geometric transform estimation or watermark detection steps. Moreover, attackers can easily remove the embedded template, because it has no secrecy. ${ }^{16}$ 
Autocorrelation function based watermarking (ACF-based watermarking) is yet another approach to resist geometric attacks. ${ }^{8,9,11}$ In this scheme, a watermark pattern is periodically embedded into an image. The periodic watermark makes periodic peaks in the autocorrelation function of the watermark. When geometric attacks are applied to the marked image, the peak pattern is also reshaped geometrically in the same way. The watermark detector uses the peak pattern to estimate the applied geometric attack. For reliable watermark detection, both the autocorrelation peaks and embedded watermark should be robust.

A common problem of the geometric attack resistant watermarking methods is that they are not sufficiently robust to geometric attacks that are combined with removal attacks. Removal attacks are those attacks, such as lossy compression and image filtering, that remove or attenuate the embedded watermark signal. Geometric attacks are often accompanied by removal attacks. Printing and scanning, one of the most popular geometric attacks, is a combination of geometric attacks and a removal attack (AD/DA conversion). Another example is lossy compression. Multimedia data are generally stored and transmitted after being compressed by using a lossy compression algorithm. Geometrically distorted images are no exception. Thus, it is desirable that watermarking schemes that are resistant to geometric attacks are also robust to removal attacks.

ACF-based watermarking is known as the most effective watermarking scheme against combined geometric-removal attacks. ${ }^{7}$ However, there is much room for improvement. As already mentioned, successful watermark detection requires that both the autocorrelation peaks and embedded watermark should survive attacks, and in commonly used ACF-based watermarking schemes, the autocorrelation peaks are not sufficiently robust. Figure 1 shows that the strength of the autocorrelation peaks is much lower than that of the watermark detector response. The autocorrelation peaks can be removed easily, which can cause watermark detection failure, even if the watermark signal remains in the host image. This means that the overall detection performance is more dependent on the autocorrelation peaks when geometric attacks have been applied to a marked image.

In this paper, we propose an improved ACF-based watermarking scheme that enhances the strength of the autocorrelation peaks and is hence more robust to geometric attacks and combined geometric-removal attacks. Conventional ACFbased watermarking methods embed watermarks simply by adding a periodic watermark to an original image. By contrast, the proposed watermarking scheme adopts watermarking with side information mechanism ${ }^{12}$ in the embedding process. The proposed embedder uses an original image and the detector information in a more sophisticated way, so that the detector can extract a clearer autocorrelation peak pattern. This improves overall detection performance against geometric attacks.

This paper is organized as follows. Section 2 describes the basic approach and detailed algorithm for the proposed method. Section 3 presents the experimental results, which show that the proposed scheme provides stronger autocorrelation 
4 C.-H. Lee, H.-K. Lee \& Y. Suh

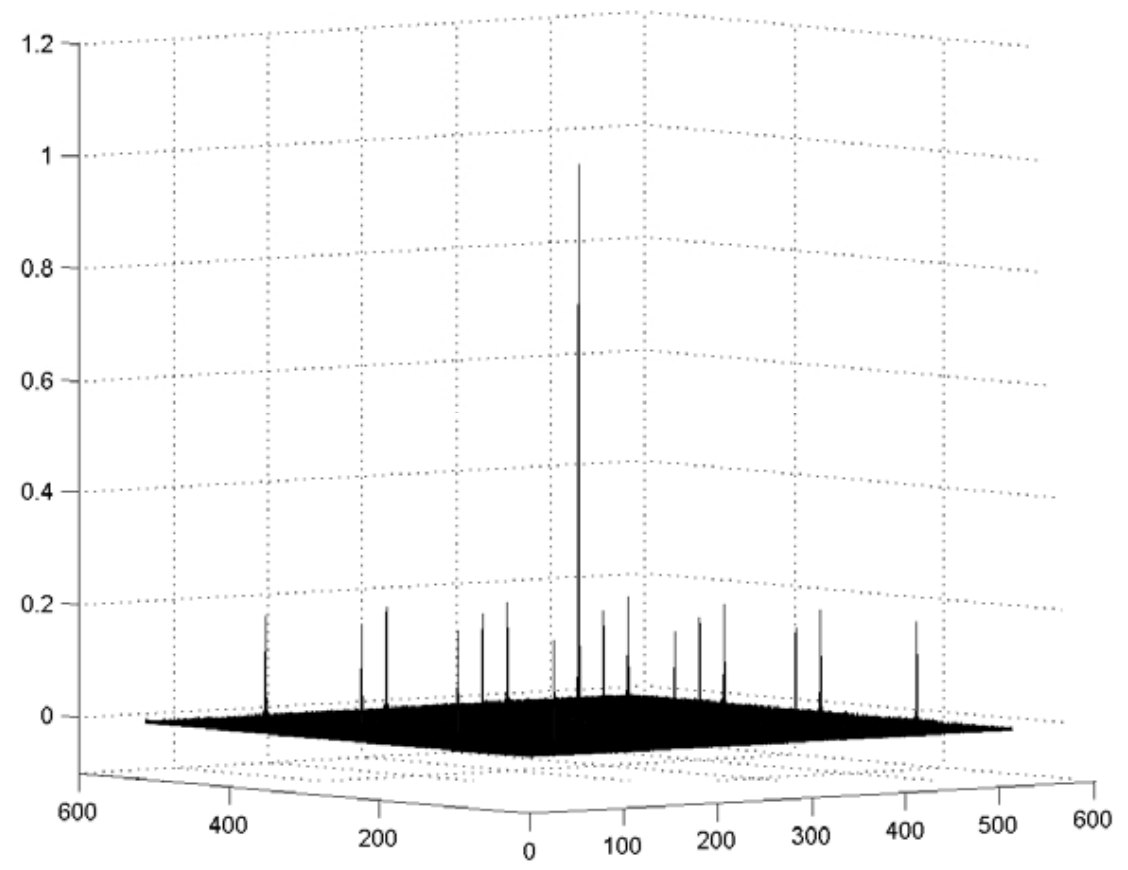

(a) Autocorrelation peaks

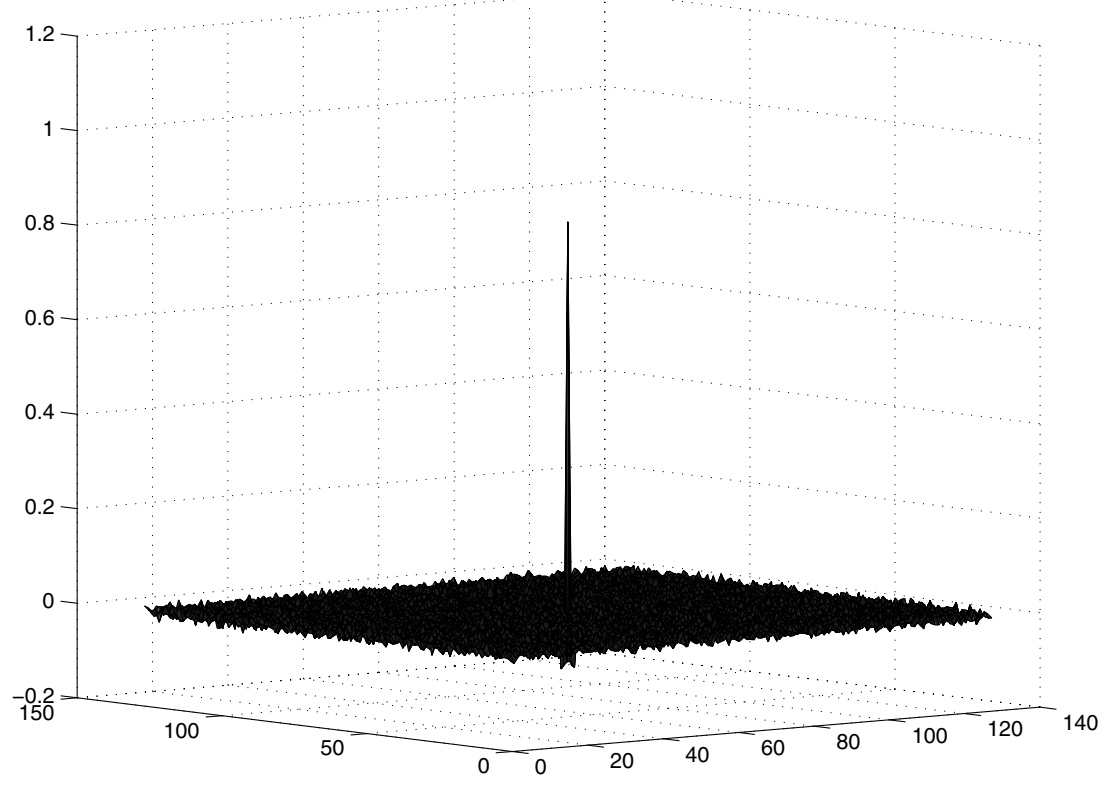

(b) Detector Response

Fig. 1. Autocorrelation peaks versus detector response. 
peaks, and hence yields better detection results, than conventional ACF-based watermarking. Concluding remarks are presented in Sec. 4.

\section{Proposed Algorithm}

\subsection{Basic approach}

5 We first explain the basic approach of the proposed scheme. At the detection process, the autocorrelation function of an extracted watermark can be modeled as

$$
\langle w+n, w+n\rangle=\langle w, w\rangle+2\langle w, n\rangle+\langle n, n\rangle,
$$

where \langle\rangle denotes the correlation operator and $w+n$ is the extracted watermark signal. $w$ is the embedded watermark signal and $n$ is the watermark estimation error of the watermark extractor. Since $n$ has no periodicity, only $\langle w, w\rangle$ is a meaningful term. The other terms are interference terms.

The proposed method improves the robustness of the autocorrelation peaks by reducing the interference introduced by $n$. To achieve this, we adopt watermarking with side information mechanism, which achieves maximum performance by exploiting an original image during the embedding process. Because we have an original image and watermark extractor information during the embedding time, we can

17 predict the estimation error $n$ and control it. Thus, by introducing periodicity to the predicted estimation error $n$ during the embedding process, we can also make $\langle n, n\rangle$ to be a meaningful term.

\subsection{Watermark embedding}

21 Figure 2 shows the proposed embedding procedure. Firstly, the watermark estimation error is predicted from an original image, by applying the watermark extractor to it. Since the original image does not contain any watermark, the extracted signal can be considered as the watermark estimation error. The extracted signal is analyzed and modified to have high autocorrelation by using its own statistical characteristics. Due to this processing, the estimation error also contains autocorrelation peaks. Finally, the processed signal is mixed with a periodic watermark and embedded into the original image.

The detailed embedding procedure is described as follows.

\subsubsection{Signal extraction}

31 A signal $E$ is extracted from an original image $I$ of size $X \times Y$ by using the watermark extractor. In the proposed scheme, the watermark extractor uses the Wiener filter ${ }^{18}$

$$
I^{-}(x, y)=\mu(x, y)+\frac{\sigma^{2}(x, y)-s^{2}}{\sigma^{2}(x, y)}(I(x, y)-\mu(x, y)),
$$




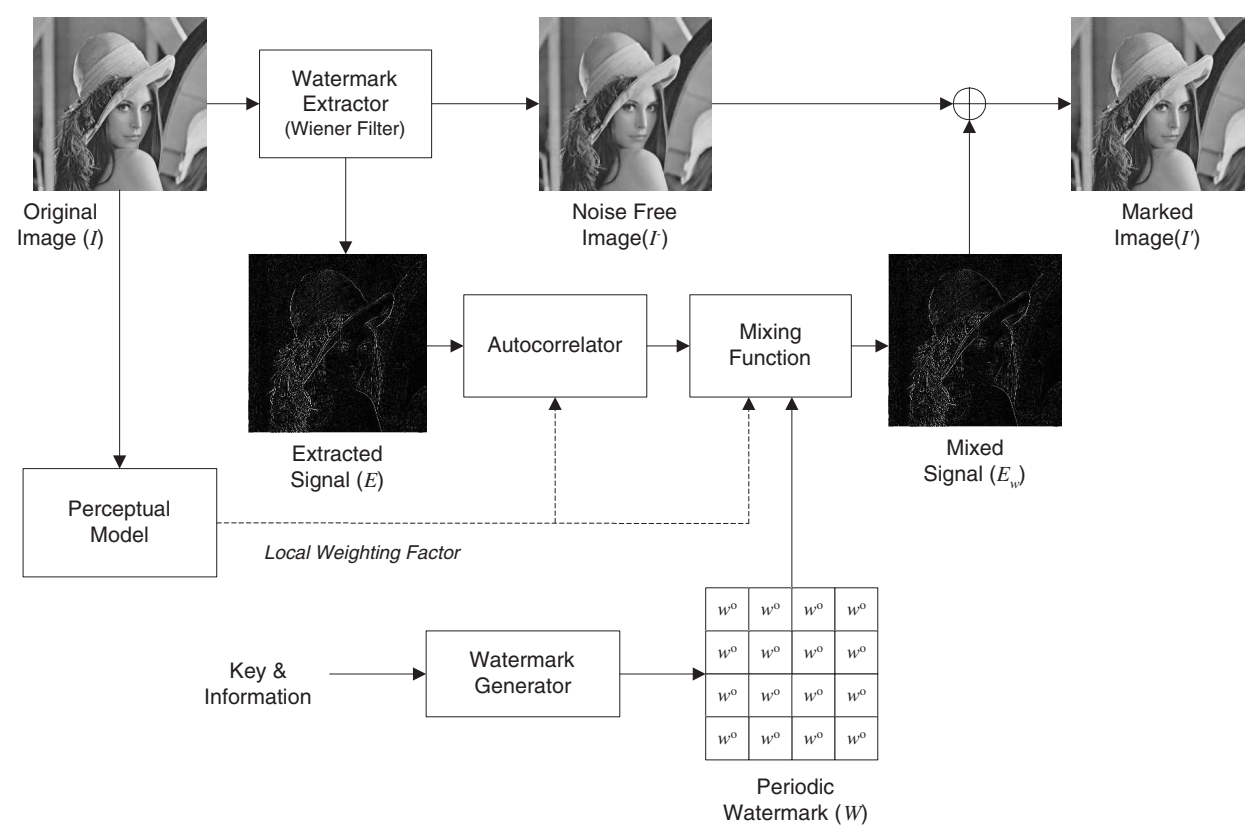

Fig. 2. Watermark embedding procedure.

where $\mu(x, y)$ and $\sigma^{2}(x, y)$ are the local mean and local variance of the original image, respectively. $s^{2}$ is the noise variance. Since the noise variance is not available, we use the average of the local variances for $s^{2}$. The extracted signal $E$ is given by

$$
E=I-I^{-}
$$

\subsubsection{Correlating process}

In the correlating process, the extracted signal $E$ is modified to have high autocorrelation with period $(M, M)$. This processing is done by segmenting the extracted signal into blocks of size $M \times M$ and modifying each block to correlate highly with the others. Figure 3 describes the geometric interpretation of this process. In the figure, each vector point represents one segmented block. In this process, a reference vector $R$ is generated first and the segmented vectors $\left(v_{1}, v_{2}, \ldots, v_{N}\right)$ are projected to new vectors $\left(v_{1}^{\prime}, v_{2}^{\prime}, \ldots, v_{N}^{\prime}\right)$ that have small angles with the reference vector $R$. This projection increases the correlation between $R$ and each vector $v_{n}^{\prime}$ :

$$
n \operatorname{Corr}\left(R, v_{n}^{\prime}\right)=\frac{R \cdot v_{n}^{\prime}}{|R|\left|v_{n}^{\prime}\right|} .
$$

As a result, the correlation between each vector is also increased. The projected vectors $\left(v_{1}^{\prime}, v_{2}^{\prime}, \ldots, v_{N}^{\prime}\right)$ construct a new signal $E^{\prime}$. Then, the signal $E^{\prime}$ has periodic high autocorrelation. 


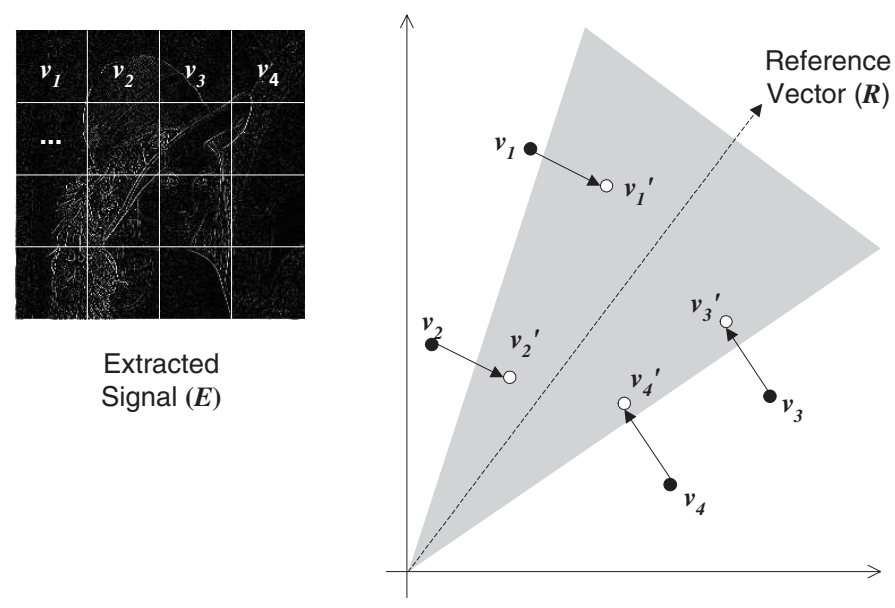

Fig. 3. Geometric interpretation of the correlating process.

To achieve high correlation with minimal distortion, the reference vector should be selected carefully. Suppose that the correlating process simply changes all segmented vectors into an identical vector to maximize the correlation. Then, the distortion will be at a minimum when the changed vector is the mean of all segmented vectors:

$$
r(i, j)=\frac{\sum_{n=1}^{N} v_{n}(i, j)}{N} .
$$

We can use this vector as the reference vector. However, the low frequency is enhanced for robustness. We enhance the low frequency by making neighboring samples have similar values. Suppose again that every segmented vector is simply changed into an identical vector in which each of the four neighboring samples has the same value. With this supposition, the distortion will be minimized when the changed vector is defined by

$$
\begin{gathered}
R(i * 2-1, j * 2-1)=R(i * 2, j * 2-1)=R(i * 2-1, j * 2)=R(i * 2, j * 2) \\
=\sum_{n=1}^{N} \frac{v_{n}(i * 2-1, j * 2-1)+v_{n}(i * 2, j * 2-1)}{+v_{n}(i * 2-1, j * 2)+v_{n}(i * 2, j * 2)} \\
4
\end{gathered}
$$

$7 \quad$ We use $R$ for the reference vector.

To ensure that each segmented vector have a small angle with the reference vector, the projection process is performed as follows. For each vector $v_{n}$, the reference vector is modified to have the same length as $v_{n}$ while maintaining the vector 11 direction. This process is described by

$$
R_{n}=\left|v_{n}\right| \cdot \frac{R}{|R|}
$$


$1 \quad$ Then, we have the difference vector between $R_{n}$ and $v_{n}$ as

$$
d_{n}=R_{n}-v_{n} .
$$

3 The segmented vector $v_{n}$ is moved close to $R_{n}$ by

$$
v_{n}^{\prime}(i, j)=v_{n}(i, j)+\alpha_{d} \lambda_{d n}(i, j) d_{n}(i, j),
$$

where $\alpha_{d}$ and $\lambda_{d n}$ are global and local weighting factors, respectively. The global weighting factor determines how much each vector is correlated with the others. The local weighing factor controls the strength of the modification of each sample adaptively by using a perceptual masking model. We use the NVF (Noise Visibility Function) based local weighting factor ${ }^{17}$ given by

$$
\lambda_{d}=(1-\mathrm{NVF}) \cdot S+\mathrm{NVF} \cdot S_{1},
$$

where $S$ and $S_{1}$ are the scaling parameter for textured and flat regions, respectively. Empirically, we set $S=3$ and $S_{1}=1 . \lambda_{d}$ is defined in the entire range of the image and $\lambda_{d n}$ is the corresponding segment of $v_{n}$ in $\lambda_{d}$. The NVF is given by

$$
\operatorname{NVF}(x, y)=\frac{1}{1+\frac{D}{\sigma_{\max }^{2}} \sigma^{2}(x, y)},
$$

where $\sigma^{2}(x, y)$ is local variance of the image and $D \in[50,100] . \sigma_{\max }^{2}$ is the maximum of the local variance.

Figure 4 shows the projection process. For simplicity, the effect of the local weighting factor is not illustrated in the figure.

\subsubsection{Watermark mixing and embedding}

The correlated signal $E^{\prime}$ is mixed with a periodic watermark and embedded into the original image. To generate a watermark signal, a basic watermark pattern $W^{\circ}$ of size $M \times M$ is generated first. For the basic watermark pattern $W^{\circ}$, a low frequency enhanced pseudo random number sequence is used. A pseudo random

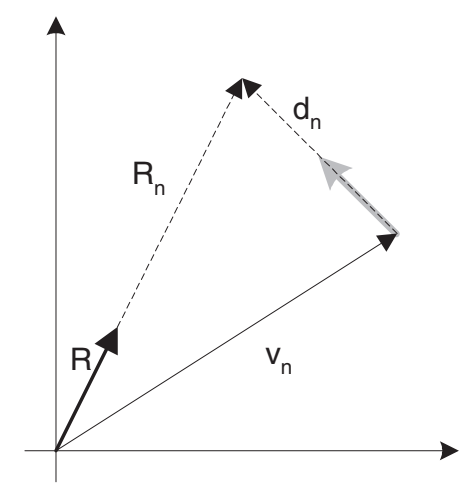

Fig. 4. Projection process for $v_{n}$. 


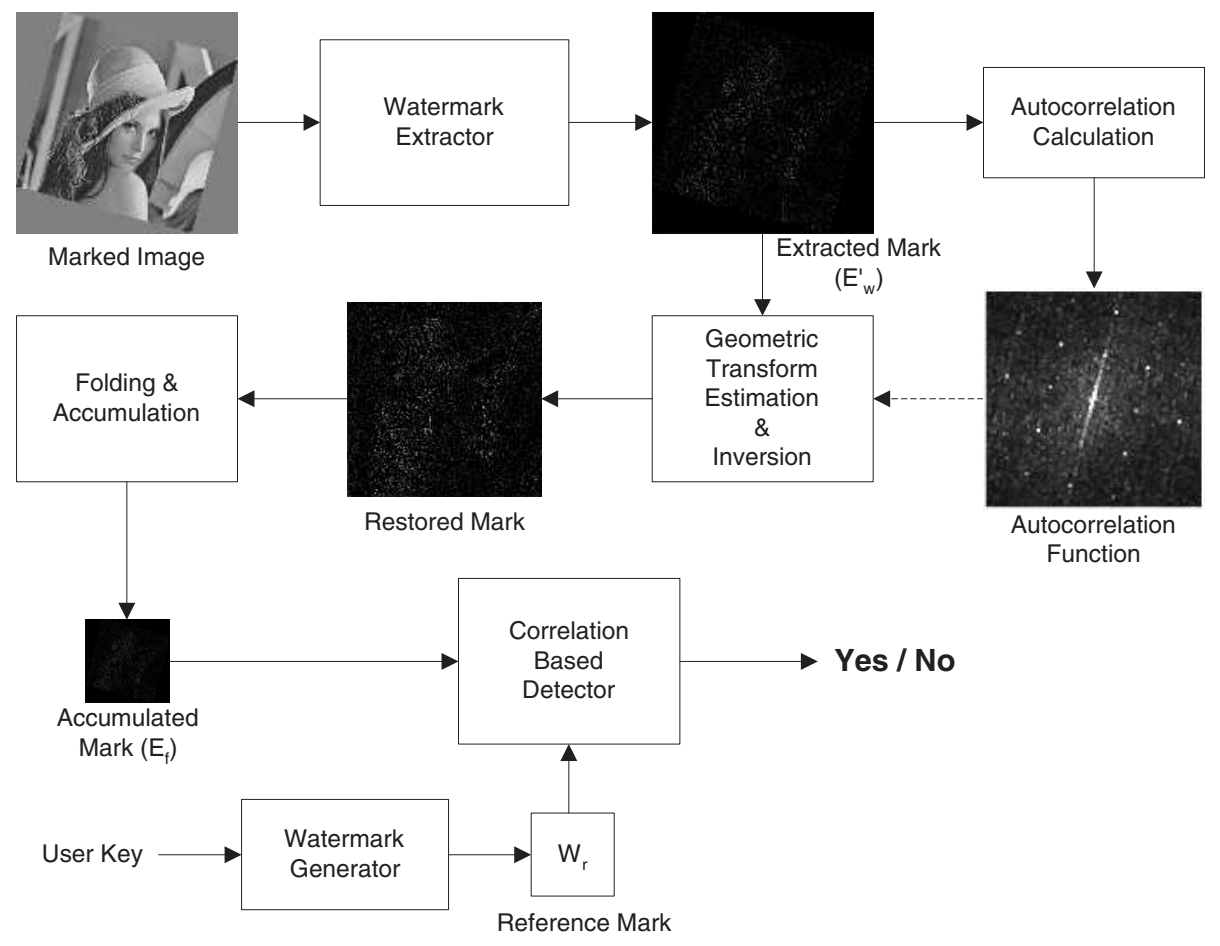

Fig. 5. Watermark detection procedure.

number sequence that follows $\mathrm{N}(0,1)$ (normal distribution with zero mean and unit variance) of size $M / 2 \times M / 2$ is generated first with a user key. The generated pattern is up-sampled into a block of size $M \times M$. The up-sampled signal is the basic watermark pattern $W^{\circ}$. We generate a periodic watermark pattern $W$ which has the same size $(X \times Y)$ as the original image by repeating the basic pattern $W^{\circ}$.

The correlated signal $E^{\prime}$ and watermark $W$ are mixed into $E_{w}$ by using a weighted summation

$$
E_{w}(x, y)=\alpha_{e} E^{\prime}(x, y)+\alpha_{w} \lambda_{w}(x, y) W(x, y),
$$

where $\alpha_{e}$ and $\alpha_{w}$ are global weighting factors and $\lambda_{w}(\cdot)$ denotes a local weighting factor that reflects perceptual masking. $\alpha_{e}$ has a value between $[0,1]$. The lower 11 the $\alpha_{e}$, the higher proportion the watermark signal occupies in the mixed signal. This will increase the detector response of the watermark, but there will be a corresponding reduction in image quality. The NVF based weighting factor is also used here for the local weighting factor $\lambda_{w}(\cdot)$.

Finally, the marked image $I^{\prime}$ is obtained by replacing the originally extracted signal $E$ in the original image $I$ with the mixed signal $E_{w}$. This operation is described as

$$
I^{\prime}(x, y)=I^{-}(x, y)+E_{w}(x, y) .
$$




\subsection{Watermark detection}

The overall detection procedure (Fig. 5) follows the general detection procedure of conventional ACF-based watermarking. The detection procedure can be divided into three processes: watermark extraction, geometric attack estimation and watermark detection.

\subsubsection{Watermark extraction}

The embedded watermark is first extracted from the marked image by using the Wiener filter, as in the embedding procedure. Generally, the host image is highly auto-correlated and has much higher energy than the watermark signal. These properties have adverse effects on watermark detection. The watermark extraction using the Wiener filter prior to the actual watermark detection reduces the adverse effects of the host images significantly. Figure 6 shows the effects of the watermark
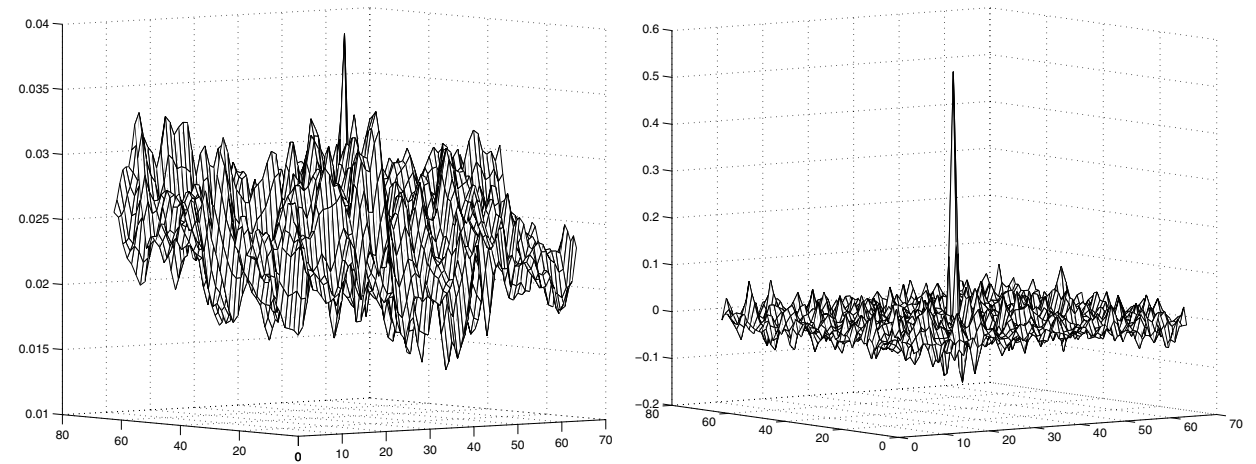

(a) Watermark detector response
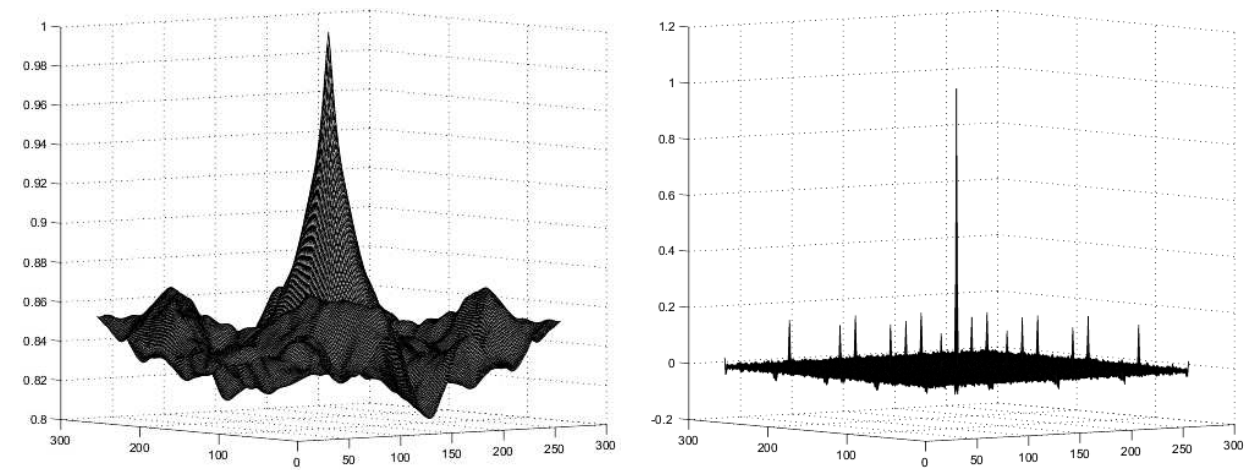

(b) Autocorrelation function

Fig. 6. Watermark detector response and autocorrelation function of a marked image (Left: without Wiener filtering, Right: with Wiener filtering). 
extraction using the Wiener filter. The extracted watermark, denoted by $E_{w}^{\prime}$, might be a corrupted version of the mixed signal $E_{w}$.

\subsubsection{Geometric attack estimation and reversion}

Prior to watermark detection, the geometric attacks applied to the marked image are estimated and reversed. Since a periodic watermark pattern was embedded into the image, periodic peaks are found in the autocorrelation function of the watermark. The geometric attacks can be estimated by using the peak pattern. The geometric attack is estimated as follows.

The autocorrelation function of the extracted watermark is calculated first. The Fast Fourier Transform (FFT) can be used for calculating the autocorrelation func-

$$
A F=\frac{\operatorname{IFFT}\left(\operatorname{FFT}\left(E_{w}^{\prime}\right) \cdot \operatorname{FFT}\left(E_{w}^{\prime}\right)^{*}\right)}{\left|E_{w}^{\prime}\right|^{2}}
$$

where "*" denotes the complex conjugate operation. If the target image contains a watermark, the autocorrelation function contains periodic peaks.

For the next step, peak detection is performed on the autocorrelation function, in two steps. First, local maximums are found in the autocorrelation function. A small window slides over the entire range of the autocorrelation function and the local maximum in each window is selected. This process removes many autocorrelation values that are not correct peaks. The window size should be determined by considering down-scaling attacks. If the window size is too big, then some correct peaks will be removed. After this preprocessing, we have some candidates for the correct peaks. In the second step, we select peaks among the candidates by

$$
A F^{\prime}(x, y)>\mu_{A F}+\alpha_{A F} \sigma_{A F},
$$

where $A F^{\prime}$ is the result of local maximum selection. $\mu_{A F}$ and $\sigma_{A F}$ denotes the average and standard deviation of the autocorrelation function respectively. $\alpha_{A F}$ is a constant.

Figure 7 shows a peak detection example. The peak detection was performed on the Lena image that had been compressed (JPEG quality factor $=50)$ and rotated by $15^{\circ}$ after the watermark embedding. As shown in the figure, the autocorrelation function has many high correlation values besides correct peaks. The proposed peak detector clearly detected the correct peaks from the autocorrelation function.

The geometric transform is estimated from the detected peaks. To explain the estimation step, we define origin peak and base peak first. The origin peak is the peak on the $(0,0)$ coordinate in the autocorrelation function. Since the normalized autocorrelation value with a signal offset of zero is always 1 , the strength of the origin peak is always 1 . The base peaks are defined as those peaks that have the distance of the watermark period from the origin peaks. For instance, if a watermark 


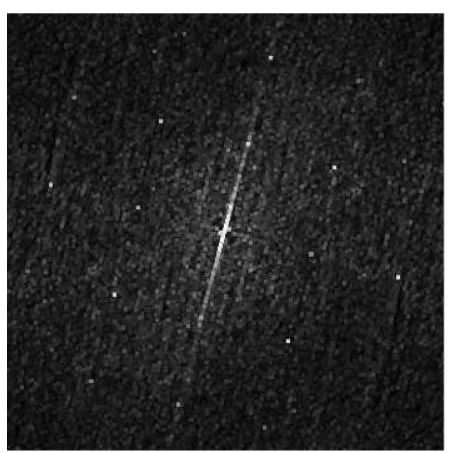

(a) Autocorrelation function of extracted mark

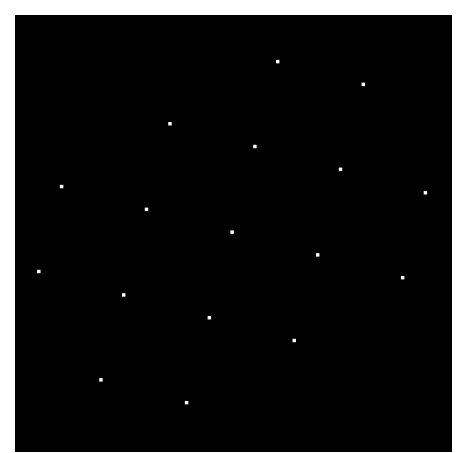

(b) Detected peaks

Fig. 7. Peak detection example from marked Lena image (JPEG compressed $(\mathrm{Q}=50)$ and $15^{\circ}$ rotated).

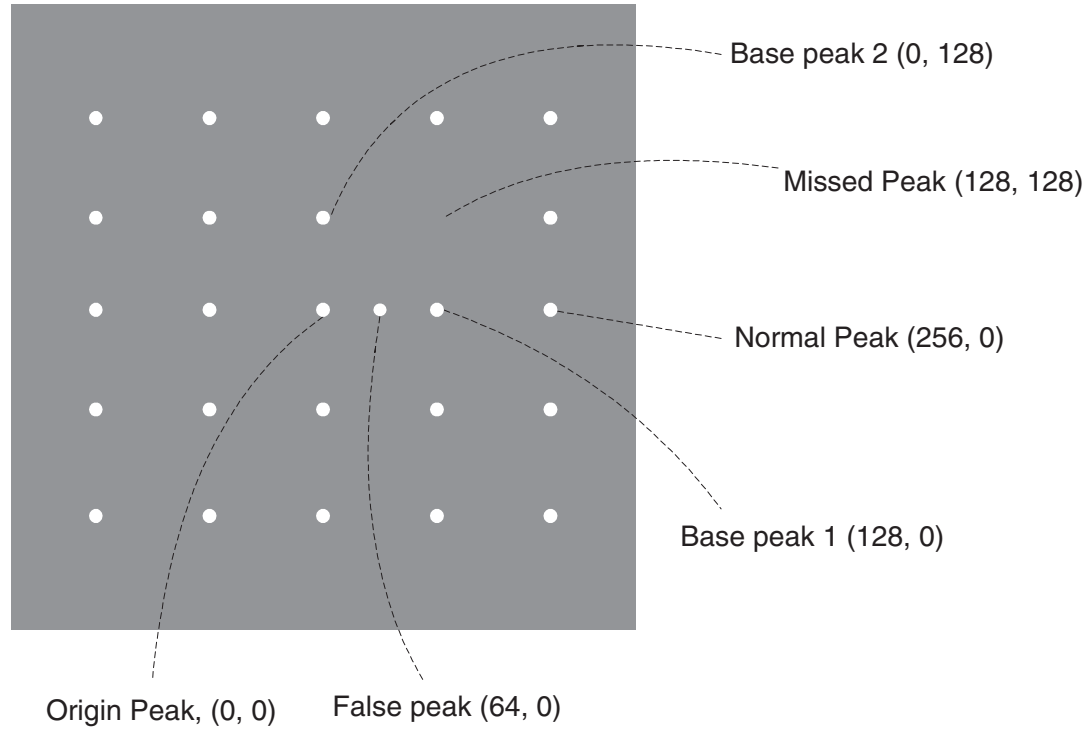

Fig. 8. Peak example for geometric transform estimation algorithm.

1 base peak coordinates are $(128,0),(0,128),(-128,0)$ and $(0,-128)$. Figure 8 shows examples of the origin peak and base peaks.

With these definitions, the distance between the origin peak and a base peak is the period of the watermark. The angle of the base peak based on the origin peak represents the rotated angle of the marked image. Thus, we can estimate the applied geometric transform by finding base peaks among the detected peaks.

The base peaks are such that if we have correct base peaks, we can find all other peaks with the offset information of the base peaks. For example, in Fig. 8, peaks 
in $(128,0)$ and $(0,128)$ are the base peaks. With the offset information of the base peaks, we can expect that there exist peaks in $(128,128),(256,0),(0,256)$ and so on. Ideally, if we can find all existing peaks with a peak, we can consider the peak as a base peak. Base peaks can be found by using this property.

Considering the aspect ratio change, we find two base peaks. If an aspect ratio change has been applied to a marked image, the base peaks in the horizontal and vertical directions have different distances from the origin peak.

Base peaks are found by the following procedures. At first, two peaks are selected from the set of detected peaks. Then we count the number of peaks in the set of detected peaks that can be found with the offset information of the currently selected peak pair. We will call this number as the "peak count" of the peak pair. This procedure is repeated for every peak pair. The peak pair with the maximum peak count can be selected as the base peak pair.

The base peak-finding method described above works well in the normal case. However, we should consider exceptional cases, which are caused by false peaks. A false peak is a peak that is detected by the peak detection method described above but is not a correct peak. Occasionally, a false peak exists between the origin and base peaks. In Fig. 8, a false peak exists on $(64,0)$. In this case, the peaks that can be found by the peak pair $[(128,0),(0,128)]$ are also found by the peak pair $[(64,0),(0,128)]$. Consequently, the peak count of peak pair $[(64,0),(0,128)]$ is higher than that of peak pair $[(128,0),(0,128)]$, so $[(64,0),(0,128)]$ is selected as the base peak pair. To avoid this situation, we consider another measure to find the base peak pair. The second measure is the ratio of the peak count to the number of the expected peak positions:

$$
\text { Peak Ratio }=\text { Peak count/Expected peak number } .
$$

In the above example, peak pair $[(64,0),(0,128)]$ has much more expected peak positions than peak pair $[(128,0),(0,128)]$. However, the peak count is only higher by one. Consequently, the peak ratio of peak pair $[(128,0),(0,128)]$ is much higher than that of peak pair $[(64,0),(0,128)]$. Thus, peak pair $[(64,0),(0,128)]$ is not selected as the base peak pair.

The peak ratio measure is also problematic. If all peaks are detected correctly in the peak detection step, the peak ratio of every peak pair is 1 . In this case, we cannot select the base peak pair. Moreover, if some normal peaks are missed, it is highly likely that an incorrect base peak pair will be selected. For example, suppose that one normal peak on $(128,128)$ is missed, as in Fig. 8 . Then, the peak ratio of peak pair $[(128,0),(0,128)]$ is lower than that of peak pair $[(128,0),(0,256)]$. As the result, the correct base peak pair is not selected as the base peak pair in this case.

To solve this problem, we use another measure given by

$$
\text { Peak Score }=\text { Peak Count } \times \text { Peak Ratio } .
$$

We can solve the exceptional cases described above with this measure. In the above examples, the peak score of the peak pair $[(128,0),(0,128)]$ is higher than those 
of peak pairs $[(64,0),(0,128)],[(128,0),(0,256)]$ and all other possible peak pairs. Finally, $[(128,0),(0,128)]$ is selected as the base peak pair in this example.

For this geometric attack estimation method to be reliable, base peaks should be more robust than other peaks. Experimentally, we found that the average strength

5

7

9 of base peaks is about $5 \%$ higher than other peaks. Therefore, we can expect this method to be reliable.

The extracted watermark signal $E_{w}^{\prime}$ is rotated and scaled to its original geometry by using the offset information of the selected base peak pair. The reversed signal is denoted by $E_{w r}^{\prime}$.

\subsubsection{Watermark detection}

The watermark detection is performed on the geometrically reversed watermark $E_{w r}^{\prime}$. Since the watermark signal is a periodic pattern with a $M \times M$ period, the restored watermark signal is segmented into blocks of size $M \times M$ and all segmented blocks are accumulated. The accumulated block is denoted by $E_{f}$. A reference watermark $W_{r}$ with size $M \times M$ is generated as in the embedding procedure with user key. The watermark detection is performed via a correlation-based detector with the accumulated signal $E_{f}$ and reference watermark $W_{r}$. To handle shift attacks, the correlation between $E_{f}$ and $W_{r}$ is calculated over all possible shifts. This process also can be carried out with reduced time complexity by using the FFT. The normalized correlation is given by

$$
Z_{n c}=\frac{\operatorname{IFFT}\left(\operatorname{FFT}\left(E_{f}\right) \cdot \operatorname{FFT}\left(W_{r}\right)^{*}\right)}{\left|E_{f}\right|\left|W_{r}\right|} .
$$

Then, the detector response is given by

$$
D R=\max _{i, j}\left\{Z_{n c}(i, j)\right\} .
$$

If $D R>\tau$, the detector decides that the watermark is detected. The threshold $\tau$ can also be decided adaptively as

$$
\tau=\mu_{z_{n c}}+\alpha_{z_{n c}} \sigma_{z_{n c}},
$$

where $\mu_{z_{n c}}$ and $\sigma_{z_{n c}}$ are the mean and standard deviation, respectively, of $Z_{n c}$.

\section{Experimental Results}

\subsection{Test environments}

To test the improvement brought about by the proposed scheme, we compared it with a conventional additive ACF-based watermarking scheme. Although current ACF-based watermarking schemes use various masking models and watermark patterns, they follow the general additive embedding model:

$$
I^{\prime}=I+\alpha \lambda W,
$$



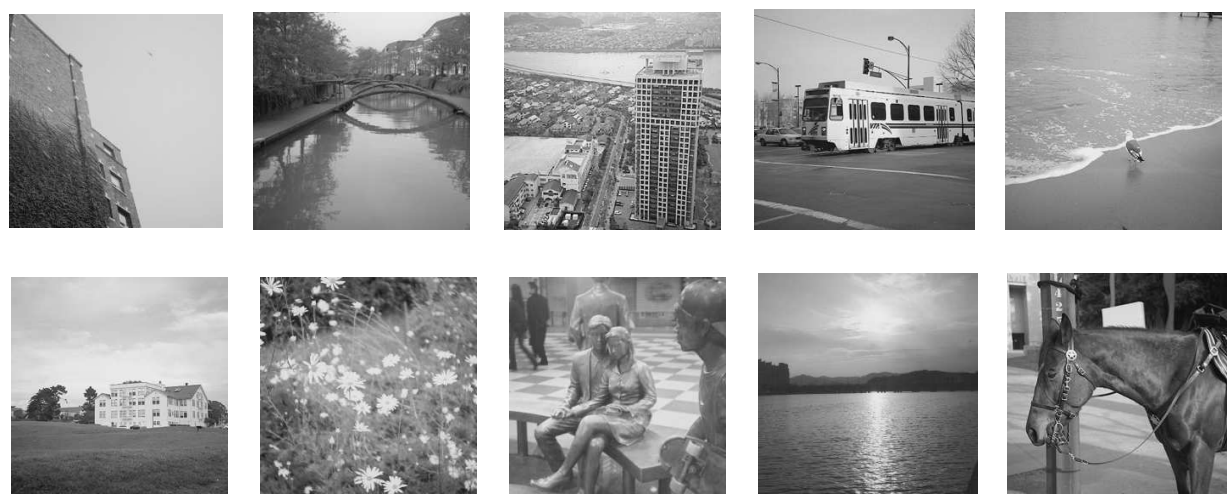

Fig. 9. Test image samples.

where $\alpha$ and $\lambda$ denote the global and local weighting factors, respectively. For a fair test environment, the same watermark pattern and local weighting factor as described in Sec. 2.2 were used for both schemes.

One thousand test images, each $256 \times 256$ pixels, were used for the experiments. Test images included natural scenes and portraits. Figure 9 shows some of test images.

The watermarking scaling factors are determined empirically. Since the correlating process also introduces distortion, the embedding strength for the actual watermark of the proposed scheme should be lower than that of the additive scheme to achieve the same level of image quality. For the additive scheme, the global scaling factor $\alpha=1.9$ in Eq. (21). For the proposed scheme, $\alpha_{w}=1.45$ and $\alpha_{e}=1$ in Eq. (12). The scaling factor $\alpha_{d}$ for the correlating process in Eq. (9) was set to $1 / \sigma_{d}$, where $\sigma_{d}$ is the standard deviation of all difference vectors $d_{n}$.

The average PSNRs of marked images of both schemes are 38 dB. Figure 10 shows the distributions of marked image quality. Both schemes show similar quality distributions.

For the watermark detection tests, we set the thresholds for autocorrelation peak and detector response as follows. For autocorrelation peak detection, we set $\alpha_{A F}=3.5$ in Eq. (15). This is a relatively low threshold and will show a high false positive rate. However, many false peaks are removed during that step in the peak detection procedure that finds local maximums and the proposed geometric transform estimation step can cancel out the effect of some false peaks. For watermark detection, we set $\alpha_{Z n c}=6$ in Eq. (20). This threshold shows about a $10^{-9}$ false positive rate theoretically.

\subsection{Statistical analysis of autocorrelation peak and detector response}

Figure 11 shows the distributions of autocorrelation peaks and watermark detector responses. Compared with the watermark detector response results, the 
16 C.-H. Lee, H.-K. Lee \&s Y. Suh

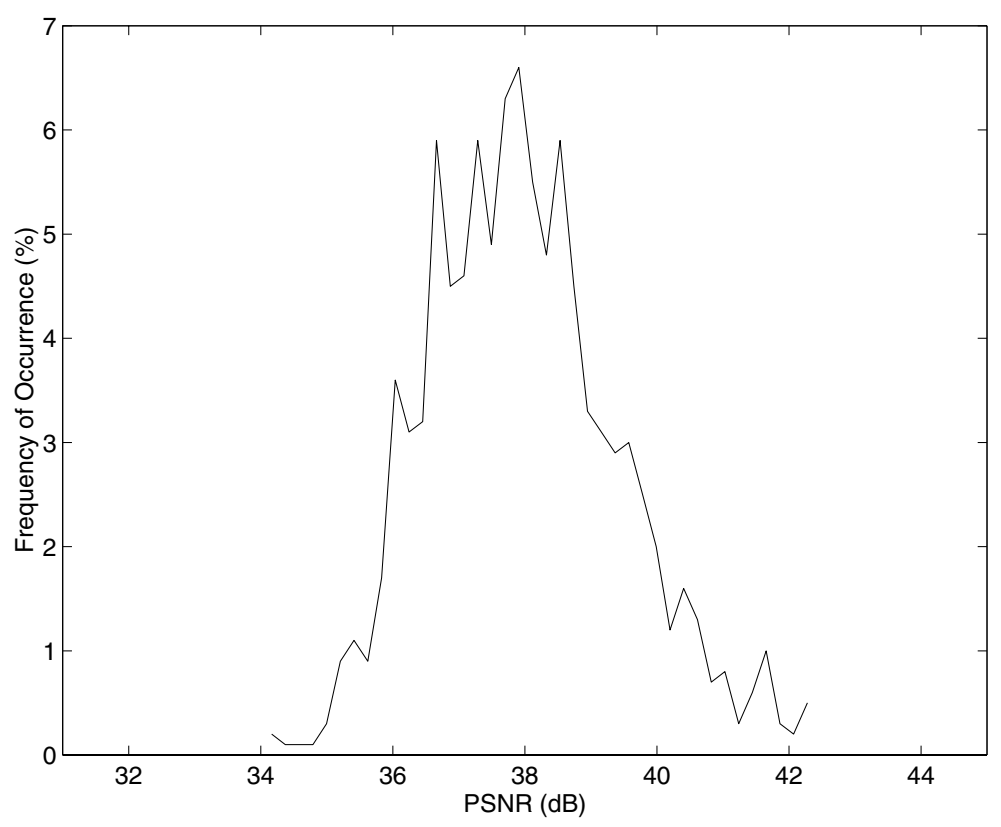

(a) Additive scheme

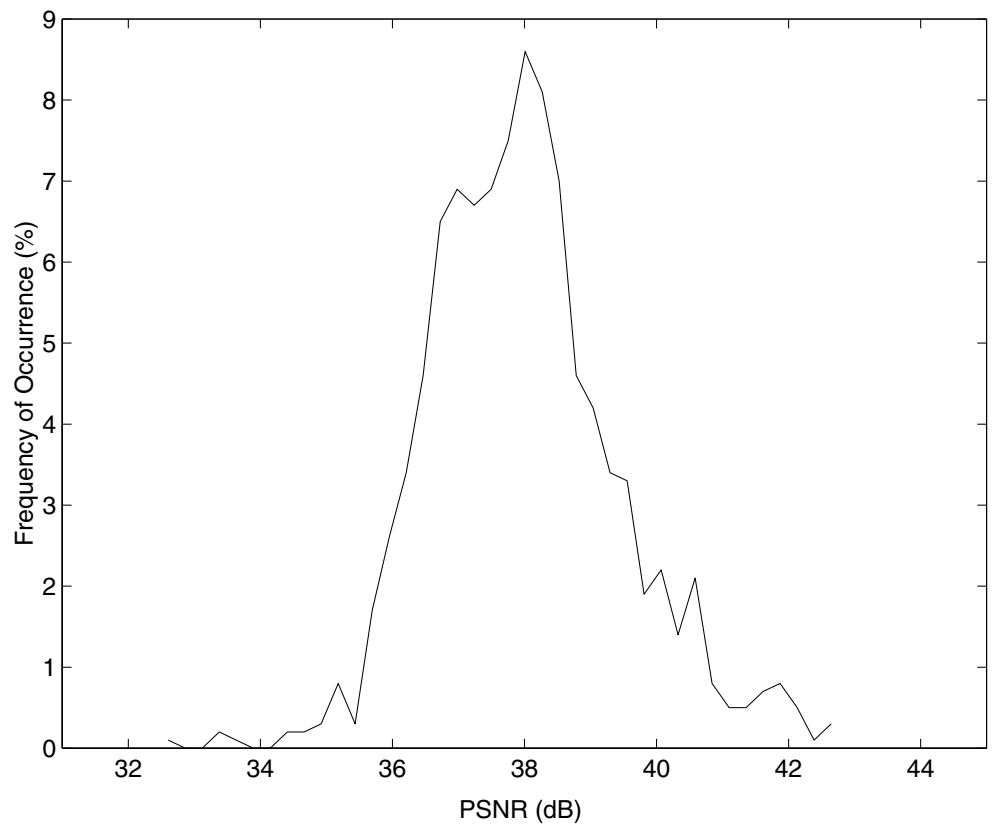

(b) Proposed scheme

Fig. 10. Distributions of quality of the marked images. 


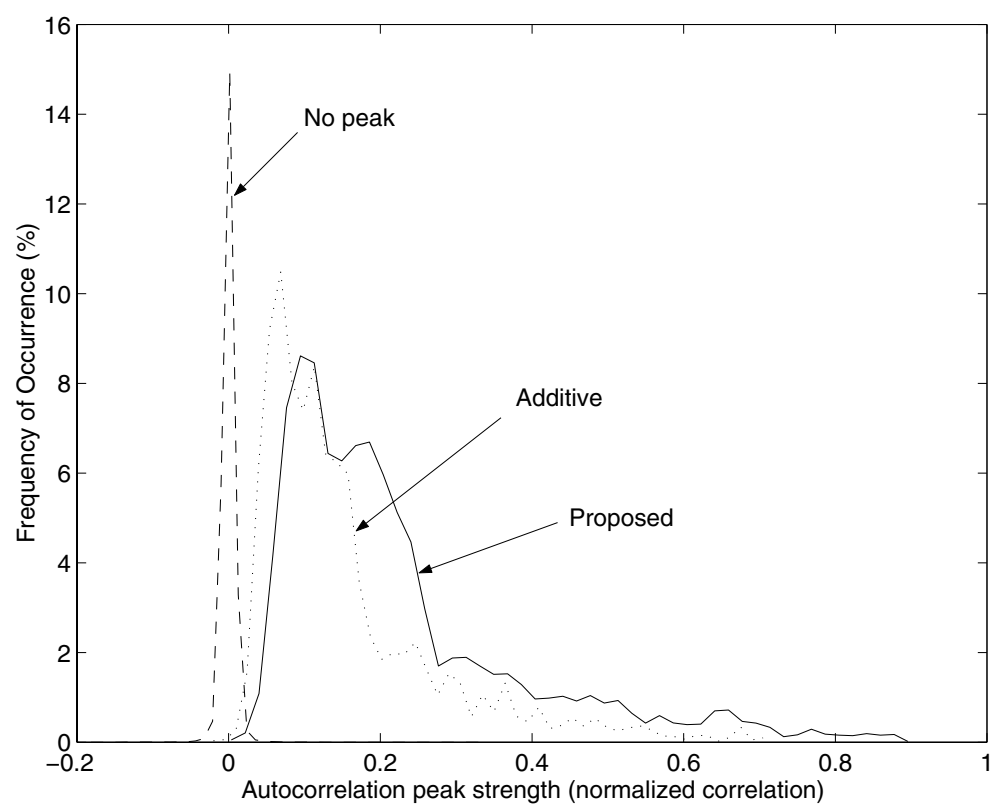

(a) Autocorrelation peak strength

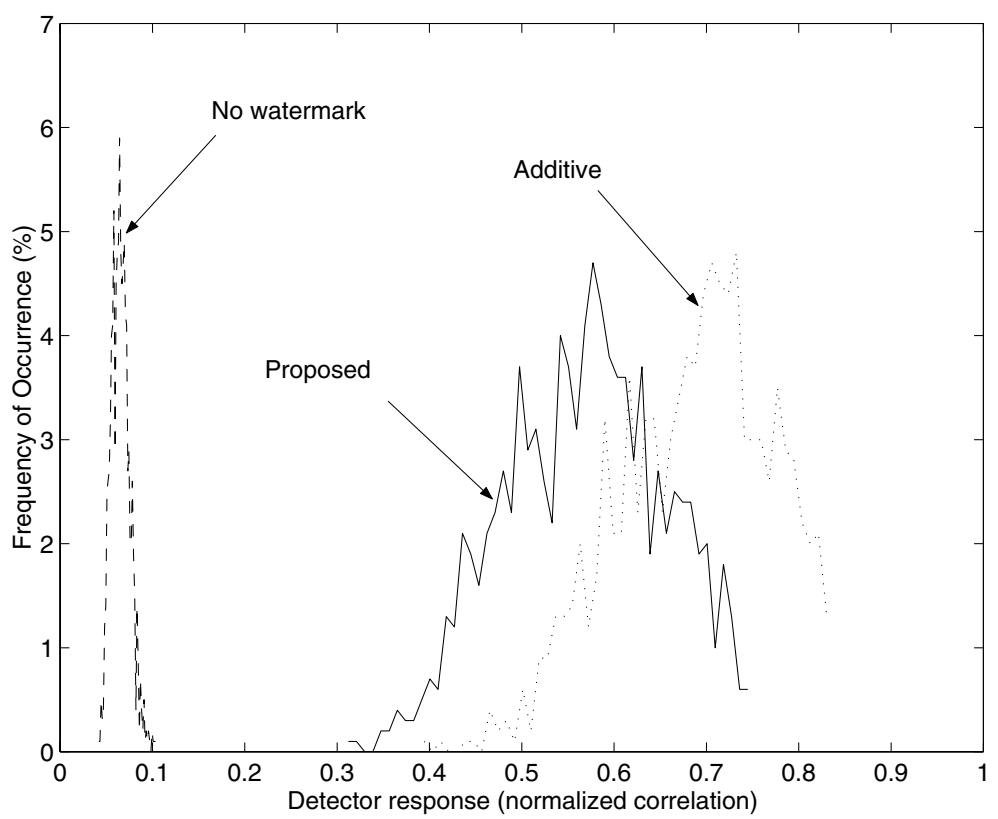

(b) Watermark detection response

Fig. 11. Distributions of autocorrelation peak strength and watermark detector response, (Average AC Peak strength $=$ [Proposed:0.2255, Additive:0.1549], Average Detector Response $=$ [Proposed:0.5680, Additive:0.6849]). 
autocorrelation peak results show poor separation between correct autocorrelation peaks and no peaks. Although the proposed scheme also does not show enough separation in autocorrelation peak distribution, the result is better than that of the additive scheme. By contrast, in the detector response results, although the proposed scheme shows relatively lower separation than the additive scheme, it is high enough for reliable watermark detection. In the figure, the average of the detector responses from unmarked images is higher than zero because the detector response is determined as the maximum correlation value between the reference mark and extracted mark with all possible shifts.

To analyze the result, we used an ROC (Receiver Operating Characteristic) curve. To get ROC curves, we should find the proper distribution model for each result. Generally, a correlation-based detector response is assumed to follow a Gaussian distribution. ${ }^{19}$ This assumption applies to the watermark detector responses in our case. However, the strength of the autocorrelation peaks does not follow a Gaussian distribution in the experiment results. Figure 12 shows this situation. In the figure, the Gaussian distribution model fits well with the distribution of the detector responses. However, the distribution of the autocorrelation peaks is not well modeled by Gaussian distribution. We found that the gamma distribution model is more appropriate for the autocorrelation peaks, as in the figure. Although it is not illustrated, watermark detector responses from unmarked images and autocorrelation values on no peak are also well modeled by Gaussian distribution. Consequently, we used the Gaussian distribution model for the watermark detector responses for marked and unmarked images and the correlation values on no peak. A Gamma distribution model was used for correct autocorrelation peak distribution.

The ROC curves and EER's (equal error rates) of autocorrelation peaks and watermark detector responses are shown in Fig. 13. For the detector responses, the proposed scheme shows relatively higher error probability than the additive scheme. However, the ROC curve and EER show that the absolute error probability is sufficiently low. On the other hand, both schemes show high probabilities of error for the autocorrelation peaks. The autocorrelation peaks show much poorer ROC curves and extremely higher EER's than the detector responses. Based on these results, if watermark detection fails for a marked image that was geometrically attacked, there is a very high probability that the error would occur in the geometric transform estimation step, because of the autocorrelation peak detection error. In this situation, the proposed scheme shows much better ROC curves and EER of the autocorrelation peaks than the additive scheme. Thus, we can expect that the proposed scheme for watermark detection will perform better than the additive scheme with respect to geometric attacks.

\subsection{Robustness of autocorrelation peak and watermark}

We tested the robustness of the autocorrelation peaks and watermarks to removal attacks. Since geometric attacks are estimated by using autocorrelation peaks, we 


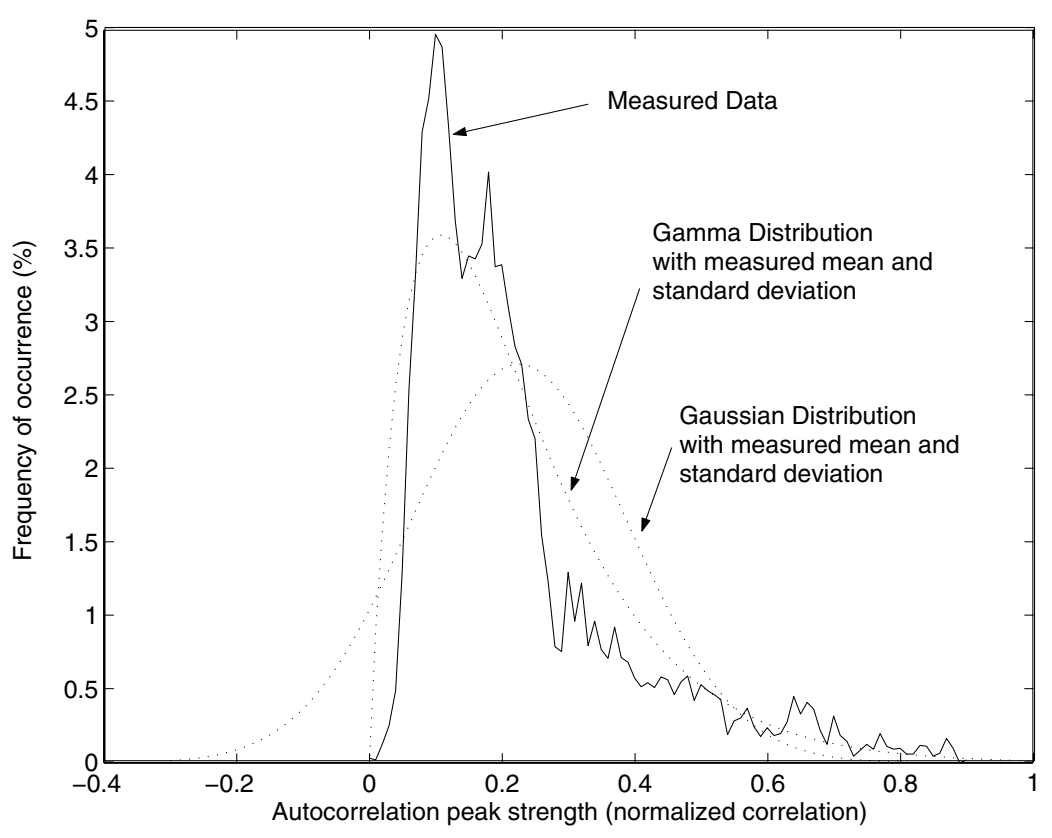

(a) AC Peaks

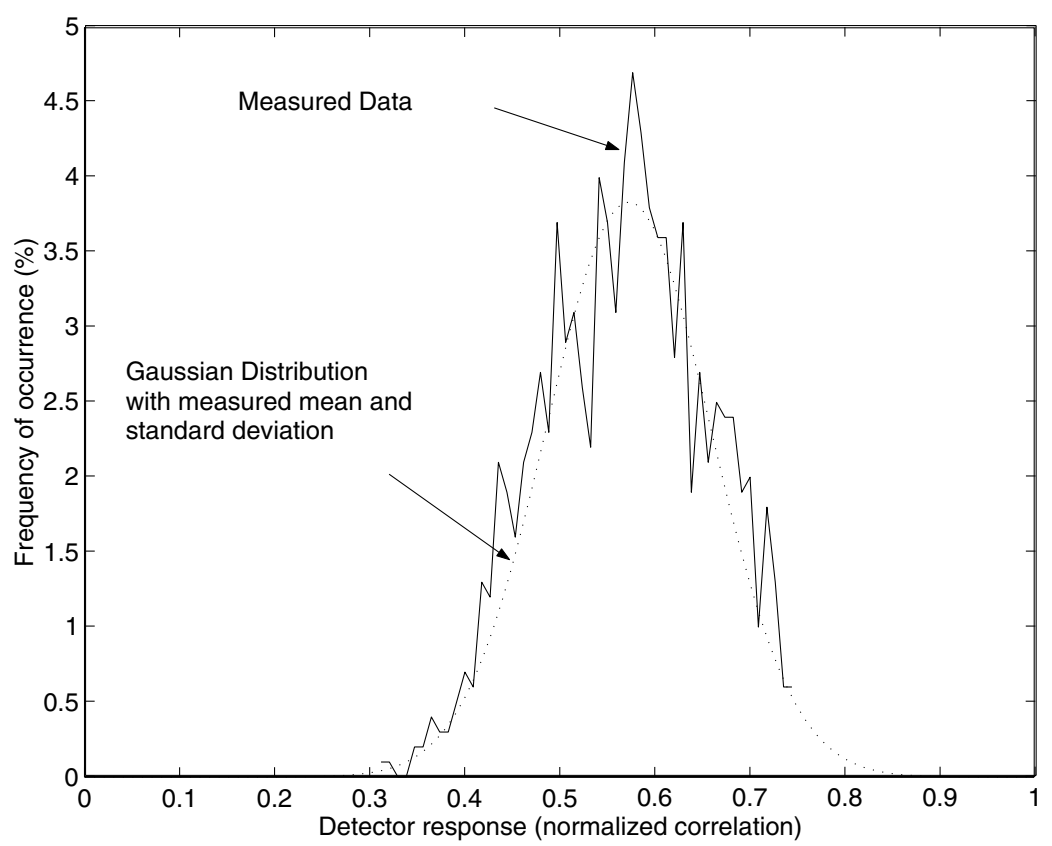

(b) Watermark detector response

Fig. 12. Distribution model for autocorrelation peak and detector response. 


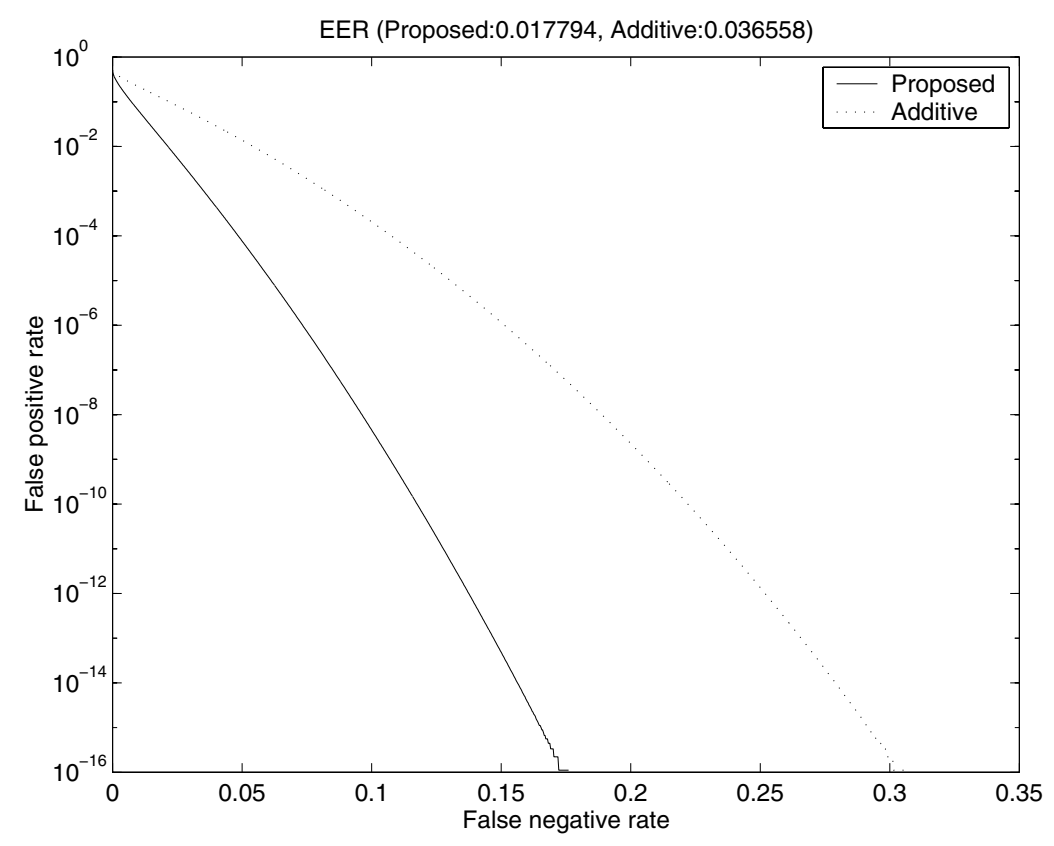

(a) AC Peaks

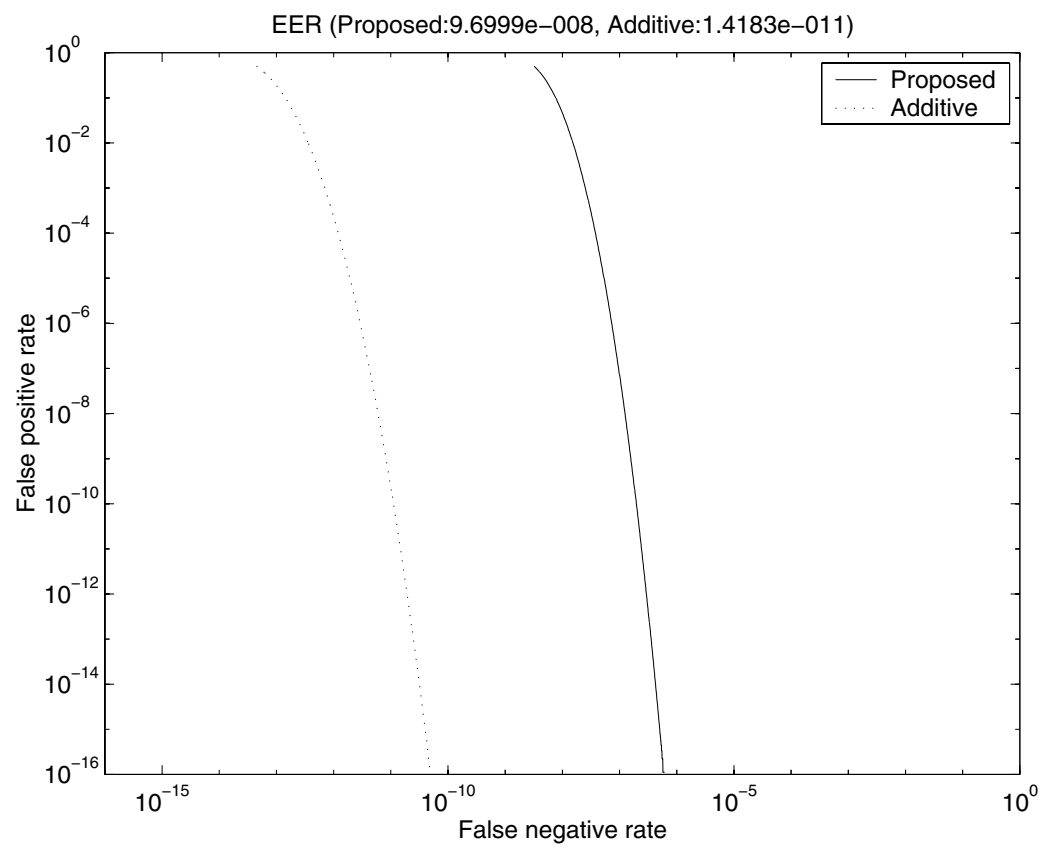

(b) Watermark detector response

Fig. 13. ROC curves and EER's of autocorrelation peak and watermark detection response. 
Image Watermarking Resistant to Geometric and Removal Attacks 21

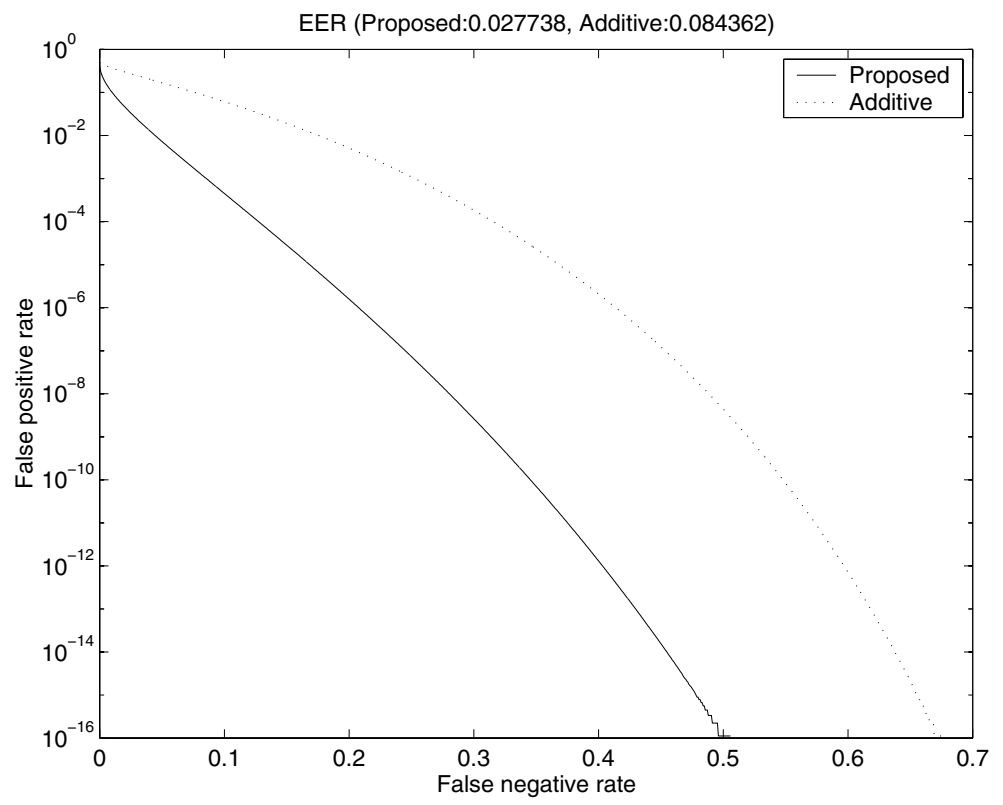

(a) AC Peaks

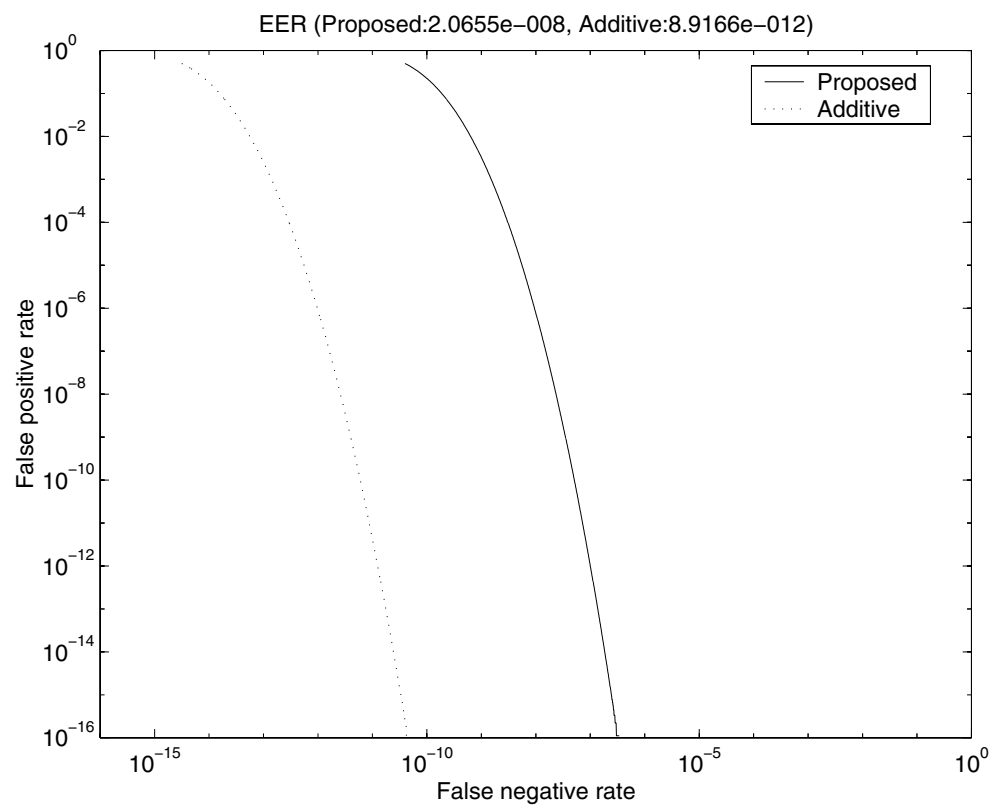

(b) Watermark detector response

Fig. 14. ROC curves and EER's after JPEG compression (Quality factor $=50 \%$ ), (Average AC peak strength $=$ [Proposed:0.0903, Additive:0.0667], Average detector response $=$ [Proposed: 0.3721, Additive:0.4911]). 
22 C.-H. Lee, H.-K. Lee \&3 Y. Suh

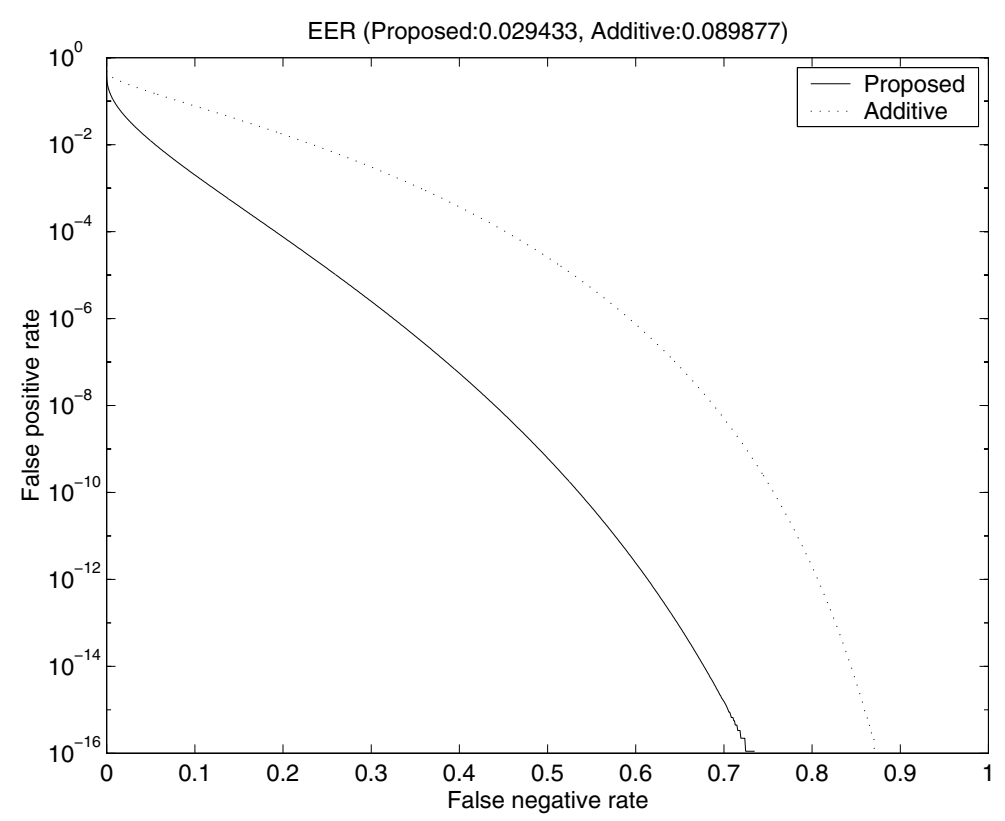

(a) AC Peaks

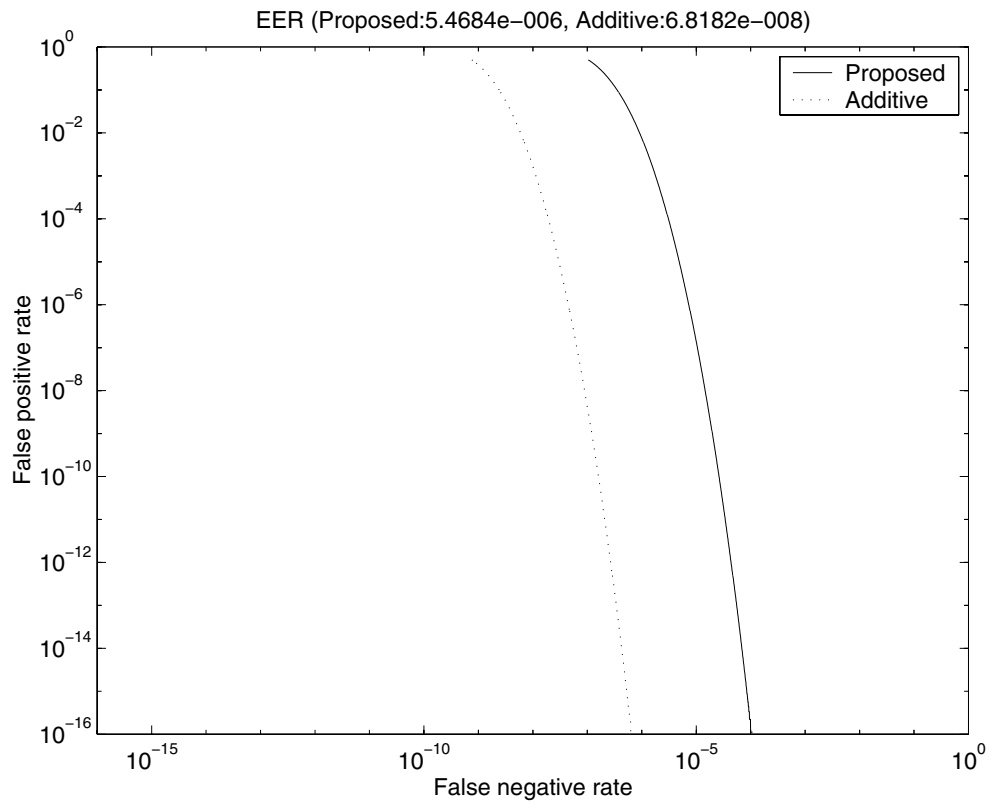

(b) Watermark detector response

Fig. 15. ROC curves and EER's after JPEG compression (Quality factor $=30 \%$ ), (Average AC peak strength $=$ [Proposed:0.0650, Additive:0.0452], Average detector response $=$ [Proposed: 0.3157, Additive:0.4228]). 


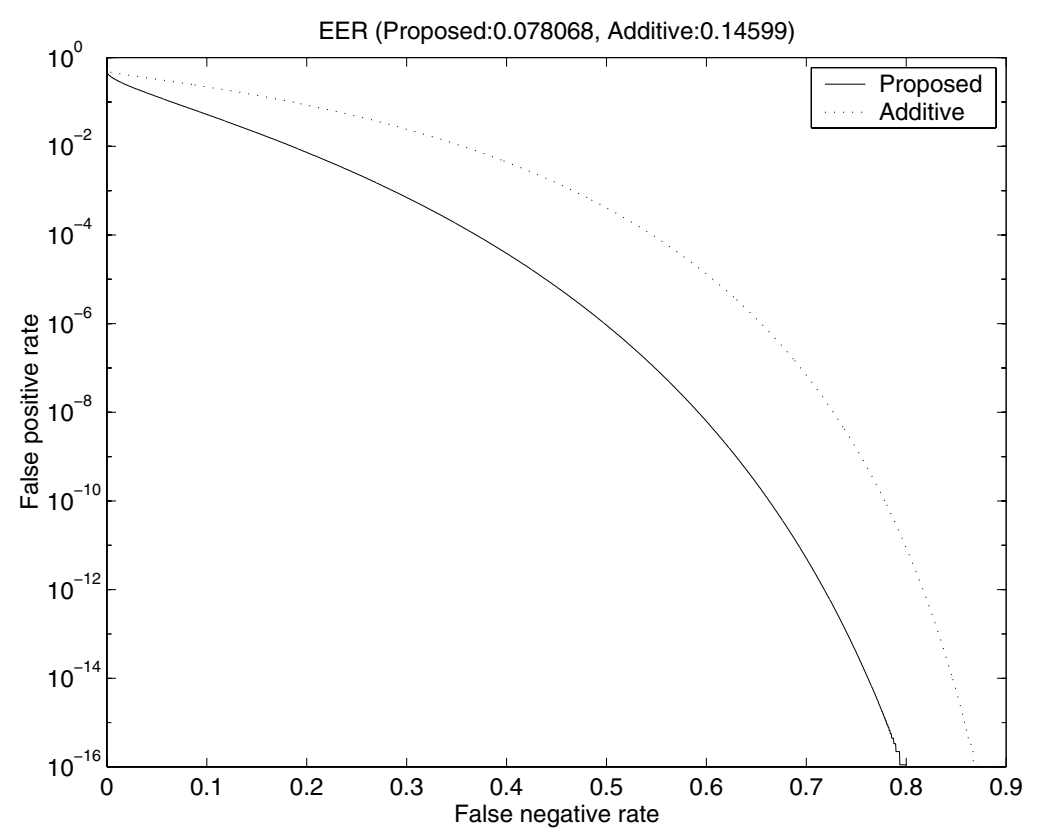

(a) AC Peaks

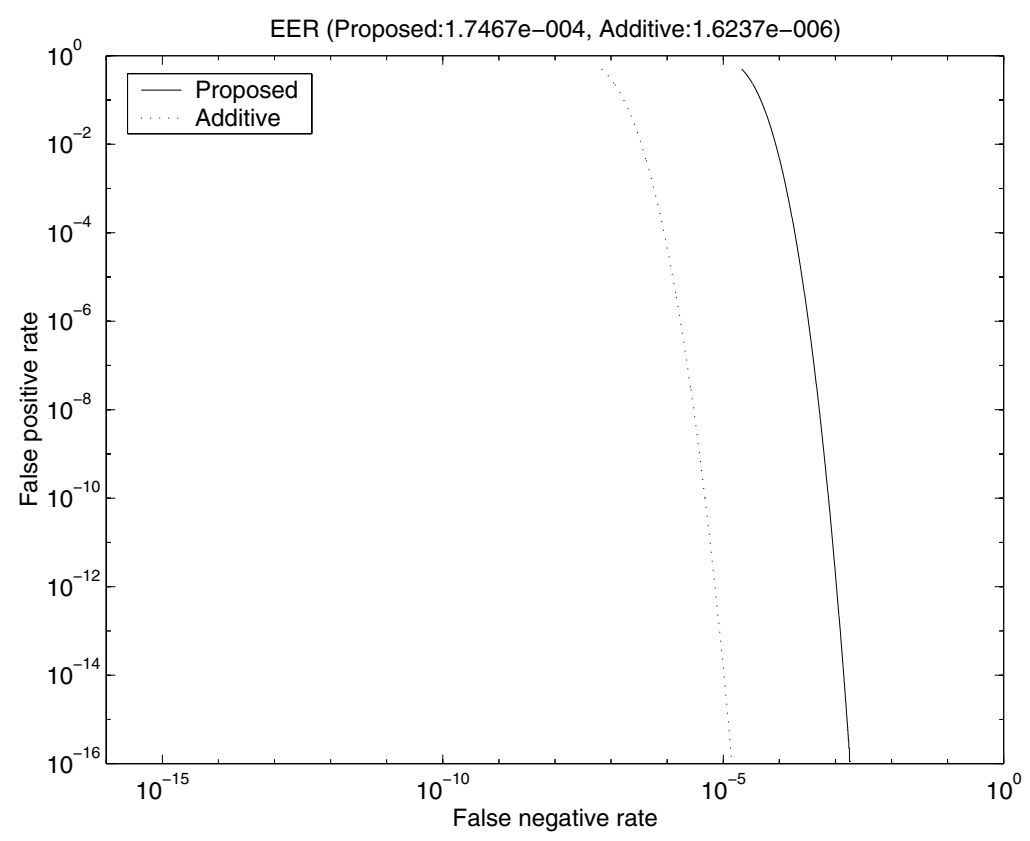

(b) Watermark detector response

Fig. 16. ROC curves and EER's after $3 \times 3$ mean filtering, (Average AC peak strength $=$ [Proposed:0.0934, Additive:0.0675], Average detector response $=$ [Proposed:0.3882, Additive:0.51]) 
can predict robustness to geometric attacks by inspecting the robustness of the autocorrelation peaks. JPEG compression (quality factor 50\% and 30\%) and $3 \times 3$ mean filtering were used for the test removal attacks.

Figures 14-16 show ROC curves and EER's after JPEG compression (quality factor $50 \%$ and $30 \%$ ) and $3 \times 3$ mean filtering. Similar to the results without attack, the proposed scheme still shows much better results for autocorrelation peak than the additive scheme for all attacks. Although the results of detector responses of the proposed scheme are not as good as the additive scheme, the probability of error is not very high. Thus, we can also expect that the proposed scheme will show better detection performance than the additive scheme against geometric-removal combined attacks.

In the measured results, the average autocorrelation peak strength and detector response after mean filtering are similar to, or a little higher than, the results after the $50 \%$ JPEG compression attack. However, the variances of the autocorrelation value on no peaks and the detector responses from unmarked images are higher than those of the JPEG compression results. The variances of peak strength and detector response for marked images are also higher. Thus, the ROC curves after mean filtering are poorer than after JPEG compression. For the same reason, the ROC curve of detector responses after JPEG 50\% compression shows better results than do ROC curves without attacks.

\subsection{Detection test results after combined attacks}

In the previous section, we observed that the proposed scheme shows improved autocorrelation peak strength over the additive scheme. Now, we test whether this achievement results in improved performance in actual detection.

For the detection test, all marked images were attacked by a geometric attack and a removal attack at the same time. The marked images were first attacked by one of the following geometric attacks.

- Rotation $\left(5^{\circ}, 15^{\circ}, 30^{\circ}\right.$ and $\left.45^{\circ}\right)$.

- Resize $(70 \%, 80 \%, 90 \%, 125 \%$ and $150 \%)$.

- Aspect ratio change $(0.7: 1,0.8: 1$ and $0.9: 1)$.

The geometrically distorted images were attacked by the following removal attacks.

- JPEG compression (Quality factor $=30$ and 50).

- Filtering $(3 \times 3$ mean filter $)$.

A watermark detection test was performed on the attacked images. It may be seen from Table 1 that the proposed scheme yields much better detection results than do the additive scheme. For most combined attacks, the proposed scheme yielded $100 \sim 200$ more successful detections. These results prove that the proposed 
Table 1. Watermark detection results after combined attacks.

\begin{tabular}{|c|c|c|c|c|}
\hline \multirow{2}{*}{$\begin{array}{c}\text { Removal } \\
\text { Attack }\end{array}$} & \multirow{2}{*}{\multicolumn{2}{|c|}{$\begin{array}{c}\text { Geometric } \\
\text { Attack }\end{array}$}} & \multicolumn{2}{|c|}{ Number of Detection } \\
\hline & & & Proposed & Additive \\
\hline \multirow{12}{*}{$\operatorname{JPEG}(\mathrm{Q}=50)$} & & $5^{\circ}$ & 553 & 147 \\
\hline & Rotation & $15^{\circ}$ & 495 & 170 \\
\hline & & $30^{\circ}$ & 845 & 644 \\
\hline & & $45^{\circ}$ & 102 & 36 \\
\hline & & $70 \%$ & 266 & 66 \\
\hline & & $80 \%$ & 451 & 135 \\
\hline & Resizing & $90 \%$ & 434 & 149 \\
\hline & & $125 \%$ & 913 & 760 \\
\hline & & $150 \%$ & 943 & 865 \\
\hline & Aspect & $0.7: 1$ & 517 & 261 \\
\hline & Ratio & $0.8: 1$ & 715 & 392 \\
\hline & Change & $0.9: 1$ & 822 & 565 \\
\hline \multirow{12}{*}{$\operatorname{JPEG}(\mathrm{Q}=30)$} & & $5^{\circ}$ & 239 & 24 \\
\hline & Rotation & $15^{\circ}$ & 273 & 62 \\
\hline & & $30^{\circ}$ & 815 & 629 \\
\hline & & $45^{\circ}$ & 17 & 5 \\
\hline & & $70 \%$ & 85 & 9 \\
\hline & & $80 \%$ & 193 & 18 \\
\hline & Resizing & $90 \%$ & 220 & 26 \\
\hline & & $125 \%$ & 839 & 548 \\
\hline & & $150 \%$ & 895 & 760 \\
\hline & Aspect & $0.7: 1$ & 315 & 106 \\
\hline & Ratio & $0.8: 1$ & 477 & 189 \\
\hline & Change & $0.9: 1$ & 658 & 298 \\
\hline \multirow{12}{*}{$\begin{array}{c}3 \times 3 \\
\text { mean filter }\end{array}$} & & $5^{\circ}$ & 508 & 259 \\
\hline & Rotation & $15^{\circ}$ & 704 & 449 \\
\hline & & $30^{\circ}$ & 771 & 577 \\
\hline & & $45^{\circ}$ & 692 & 494 \\
\hline & & $70 \%$ & 276 & 157 \\
\hline & & $80 \%$ & 464 & 262 \\
\hline & Resizing & $90 \%$ & 305 & 157 \\
\hline & & $125 \%$ & 873 & 695 \\
\hline & & $150 \%$ & 924 & 810 \\
\hline & Aspect & $0.7: 1$ & 360 & 194 \\
\hline & Ratio & $0.8: 1$ & 589 & 335 \\
\hline & Change & $0.9: 1$ & 659 & 392 \\
\hline
\end{tabular}

1 embedding scheme improves the robustness of ACF-based watermarking against combined geometric-removal attacks.

3 Although the poorest ROC curves are observed after the mean filter attack, the detection results after mean filtering show a level similar to JPEG compression 
with quality factor 50 . As mentioned in the previous section, the measured average peak strength and detector response after mean filtering are similar to, or a little higher than after, JPEG compression. We suppose this is one reason for better detection results. Another reason is supposed to be the block-based compression of JPEG. The blocking artifacts after geometric attacks might have a bad effect on autocorrelation peak detection.

\section{Conclusion}

No previous watermarking scheme has sufficient robustness against geometric attacks that are combined with removal attacks. ACF-based watermarking is known to be the most effective against combined attacks. However, ACF-based watermarking has two detection failure modes and the autocorrelation peaks are not sufficiently strong. The proposed method provides greater robustness to the combined attacks by enhancing the strength of the autocorrelation peaks of ACF-based watermarking. By sophisticated use of the information of an original image and the detector structure at the time of embedding, strong autocorrelation peaks are obtained at the time of detection. Experimental results showed that the proposed method yielded stronger autocorrelation peaks than the conventional ACF-based method. As a result, the proposed scheme showed better detection results for various combined attacks than conventional ACF-based watermarking.

Future work will focus on improving the watermark detector response. Although the proposed scheme improved the robustness to the geometric attacks by enhancing the autocorrelation peak strength, the watermark detector response was reduced slightly. We will research more about this problem in future work.

Another problem with ACF-based watermarking is the lack of security at the geometric transform estimation step. Key-based security is only adopted at the final watermark detection step. Since the watermark estimator is known publicly, anyone can extract the autocorrelation peak pattern and guess the residence of a watermark in an image. An intentional attacker can estimate the embedded watermark by using the peak pattern and statistical characteristics of the extracted watermark. Attackers can also remove the autocorrelation peak by de-correlating the estimated watermark. The proposed method inherits these problems from conventional ACFbased watermarking, which remain to be solved in future work.

\section{References}

1. N. Memon and P. W. Wong, "Protecting digital media content," Communications of the ACM 41(7), 35-43 (July 1998).

2. G. C. Langelaar, I. Setyawan and R. L. Lagendijk, "Watermarking digital image and video data: A state-of-the-art overview," IEEE Signal Processing Magazine 17(5), 20-46 (September 2000).

3. I. J. Cox, J. Kilian, F. T. Leighton and T. Shamoon, "Secure spread spectrum watermarking for multimedia," IEEE Transactions on Image Processing 6(12), 1673-1687 (1997). 
4. S. Pereira, J. J. K. O. Ruanaidh, F. Deguillaume, G. Csurka and T. Pun, "Template based recovery of Fourier-based watermarks using log-polar and log-log maps," IEEE International Conference on Multimedia Computing and Systems 1, 870-874 (1999).

5. J. J. K. O'Ruanaidh and T. Pun, "Rotation, scale and translation invariant spread spectrum digital image watermarking," Signal Processing 66(3), 303-317 (May 1998).

6. S. Pereira and T. Pun, "Fast robust template matching for affine resistant image watermarking," In International Workshop on Information Hiding, Vol. LNCS 1768 of Lecture Notes in Computer Science, pp. 200-210 (October 1999).

7. S. Voloshynovskiy, F. Deguillaume and Thierry Pun, "Content adaptive watermarking based on a stochastic multiresolution image modeling," In Tenth European Signal Processing Conference (EUSIPCO'2000), Tampere, Finland (September 5-8, 2000).

8. M. Kutter, "Watermarking resisting to translation, rotation, and scaling," In SPIE International Symposium on Voice, Video, and Data Communications 3528, 423-431, Boston, USA (November 1998).

9. P.-C. Su and C.-C. J. Kuo, "Synchronized detection of the block-based watermark with invisible grid embedding," Proc. of SPIE, Security and Watermarking of Multimedia Contents III 4314, 406-417, San Jose, USA (January 2001).

10. C.-Y. Lin, M. Wu, J. A. Bloom, I. J. Cox, M. L. Miller and L. M. Yui, "Rotation, scale, and translation resilient watermarking for images," IEEE Trans. on Image Processing 10(5), 767-782 (May 2001).

11. S. Voloshynovskiy, F. Deguillaume and T. Pun, "Multibit digital watermarking robust against local nonlinear geometric distortions," 2001 IEEE Int. Conf. of Image Processing (ICIP 2001) 3, 999-1002, Thessaloniki, Greece (October 2001).

12. I. J. Cox, M. L. Miller and A. L. McKellips, "Watermarking as communications with side information," Proceedings of the IEEE 87(7), 1127-1141 (July 1999).

13. M. Kutter, S. K. Bhattacharjee and T. Ebrahimi, "Towards second generation watermarking schemes," IEEE Int. Conf. of Image Processing 1999 1, 320-323, Kobe, Japan (October 25-28, 1999).

14. P. Bas and J.-M. Chassery, "Robust watermarking based on the warping of predefined triangular patterns," Proceedings of SPIE, Security and Watermarking of Multimedia Contents II 3971, 99-109, San Jose, USA (January 2000).

15. F. Hartung and B. Girod, "Watermarking of uncompressed and compressed video," Signal Processing 66(3), 283-301 (May 1998).

16. A. Herrigel, S. Voloshynovskiy and Y. Rytsar, "The watermark template attack," Proceedings of the SPIE Security and Watermarking of Multimedia Contents III 4314, 394-405, San Jose, USA (January 22-25, 2001).

17. S. Voloshynovskiy, A. Herrigel, N. Baumgartner and T. Pun, "A stochastic approach to content adaptive digital image watermarking," In International Workshop on Information Hiding, Vol. LNCS 1768 of Lecture Notes in Computer Science, pp. 212-236, Springer Verlag, Dresden, Germany (1999).

18. J. S. Lim, Two-Dimensional Signal and Image Processing, Prentice Hall, USA (1990).

19. I. J. Cox, M. L. Miller and J. A. Bloom, Digital Watermarking, Academic Press (2002). 

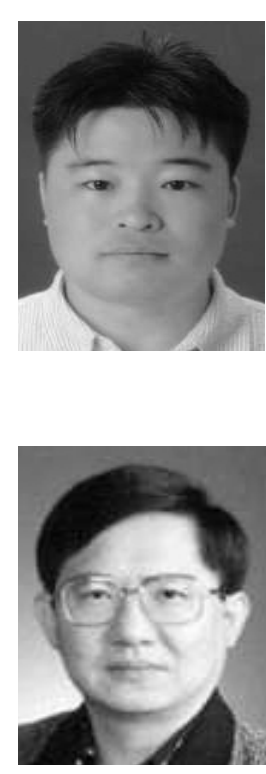

Choong-Hoon Lee received the BE degree in computer engineering from Dongguk University, Seoul, Korea, in 1996, and the MS degree in computer science from Korea Advanced Institute of Science and Technology (KAIST), Daejon, Korea, in 1998, respectively.

Currently he is a $\mathrm{PhD}$ candidate in computer science at KAIST. His research interests are digital watermarking, DRM (Digital Right Management), and image and video coding.

Heung-Kyu Lee received the BS degree in electronics engineering from the Seoul National University, Seoul, Korea, in 1978, and the MS, PhD Degrees in computer science from the Korea Advanced Institute of Science and Technology(KAIST), in 1981, and 1984, respectively.

From 1984 to 1985 he served as a research scientist at the University of Michigan, Ann Arbor. Since 1986 he has been a professor in the Department of Computer Science, the Korea Advanced Institute of Science and Technology, Daejon, Korea 305701(hklee@casaturn.kaist.ac.kr). He received the Order of Civil Merit, SukRyu medal 5066 from the Republic of Korea in October 1992. He was a director of the Korean Society of Remote Sensing. He is now a director of the DRM (Digital Right Management) forum and a director for the international affair of Korea Institute of Information Security and Cryptology. He is also vice director of the AITRC center in the KAIST.

He is an author/coauthor of more than 100 journal and conference proceedings papers in his research areas. He has been a reviewer of many international journals, J. of Electronic Imaging, Real-Time Imaging, ACM Multimedia System - Journal, etc. He was a program chairman of IEEE real-time computing systems and applications in 1999 and 2000, respectively. His biography has been selected by Marquis Who's Who for inclusion in Who's Who in Science and Engineering, Who's Who in America, and Who's Who in the World since 1996.

His major interests are digital watermarking, fingerprinting, and DRM (Digital Right Management). 


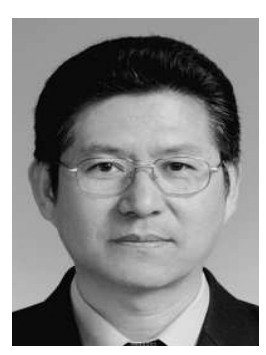

Youngho Suh received the BS degree in computer science from Kyungpook National University, Taegu, Korea, in 1979, and the MS degree in computer science from Kwangwoon University, Seoul, Korea, in 1995. Currently he is a PhD candidate in computer science at Chungnam National University. From 1979 to 1998 he served as a principal researcher at the Systems Engineering Research and Institute (SERI), Daejeon, KOREA.

Since 1998 he has been a team leader in the Digital Content Research Division, Electronics and Telecommunications Research Institute (ETRI), Daejeon, Korea 305-350(syh@etri.re.kr).

He is now a director of the DRM (Digital Right Management) forum in Korea. His research interests include digital watermarking, fingerprinting, and DRM. 